This is the authors' post-print copy of a manuscript submitted to Pharmacological Research.

Please cite as:

Arroo Randolph R.J. \& Alfa Hajara H. (2018) Chemical properties of thymoquinone, a monoterpene isolated from the seeds of Nigella sativa Linn. Pharmacological Research 133: 151

https://doi.org/10.1016/j.phrs.2018.05.003 


\section{Chemical properties of thymoquinone, a monoterpene isolated from the seeds of Nigella sativa}

Linn.

Randolph R.J. Arroo, Hajara H. Alfa

Leicester School of Pharmacy, De Montfort University, The Gateway, Leicester, LE1 9BH, United

Kingdom.E-mail address: rrjarroo@dmu.ac.uk

Thymoquinone is the main ingredient of the essential oil extracted from the seeds of Nigella sativa $\mathrm{L}$. (Ranunculaceae). The monoterpene is considered to be the active pharmaceutical ingredient in the seeds, which have traditionally been highly prized for their medicinal properties. The compound has been the focus of a considerable number of pharmacological investigations and has been reviewed regularly for its action against a variety of inflammatory diseases, its effect on metabolic syndrome, and its potential anticancer properties.

While discussing the chemical and pharmacological properties of thymoquinone, recent reviews have reflected on the keto-enol tautomerism of thymoquinone. Specifically, thymoquinone is described as a tautomeric compound, where the keto-form is said to be the major configuration that is responsible for its pharmacological properties $[1,2]$. In both reviews, reference is made to a 2005 review by Salem [3]. The latter review discusses a range of activities of thymoquinone, mainly on cell signalling and antioxidant (scavenging) molecular mediators involved in the process of inflammation. However, no mention is made in this review of keto- or enol forms of thymoquinone. Moreover, the chemical structure of thymoquinone does not allow keto-enol tautomerism.

The confusion might have been introduced in 2011 by Alkharfy et al. [4] who state in the introduction of their paper that thymoquinone exists in tautomeric forms including the enol form, the keto form and mixtures. The keto form is said to be the major fraction ( $90 \%)$ as evident by thin layer chromatography (TLC) and high performance liquid chromatography (HPLC) analyses, and is 
held responsible for the pharmacological properties of the compound. A figure is included in the paper, purporting to show the tautomeric keto- and enol-forms of thymoquinone, but in reality showing thymoquinone $\left(\mathrm{C}_{10} \mathrm{H}_{12} \mathrm{O}_{2}\right)$ and its reduced form thymohydroquinone $\left(\mathrm{C}_{10} \mathrm{H}_{14} \mathrm{O}_{2}\right)$. No further reference is given for the observations, so we must assume that these are the authors' own results. The next sentence in the paper refers to the pharmacological properties of thymoquinone, and mentions that it has been studied for its cardiovascular, respiratory, cytotoxic, anti-inflammatory, and immunomodulatory properties, with reference to Salem's 2005 review [3].

Thus, tautomerism of thymoquinone was suggested by Alkharfy et al. [4], and the authors might be cited for that, although it was based on a misinterpretation. Reference of thymoquinone tautomerism to Salem $[1,2]$, is in turn based on a further misinterpretation of the text in Alkharfy's introduction [4].

Ironically, a mechanism of action has been proposed which involves intracellular non-enzymatic metabolic activation of thymoquinone by GSH, NADH or NADPH, which represents a 'cellular switch' able to modulate cellular antioxidant defences. In this model, the enol thymohydroquinone is the more active antioxidant scavenger [5].

\section{References}

[1] M.K. Shanmugam, F. Arfuso, A.P. Kumar, L. Wang, B.C. Goh, K.S. Ahn, A. Bishayee, G. Sethi, Modulation of diverse oncogenic transcription factors by thymoquinone, an essential oil compound isolated from the seeds of Nigella sativa Linn., Pharmacol.Res. 129 (2018) 357-364. DOI: 10.1016/j.phrs.2017.11.023

[2] S. Darakhshan, A. Bidmeshki Pour, A. Hosseinzadeh Colagar, S.Sisakhtnezhad, Thymoquinone and its therapeutic potentials, Pharmacol.Res. 95-96 (2015) 138-158. DOI:

10.1016/j.phrs.2015.03.011 
[3] M.L. Salem, Immunomodulatory and therapeutic properties of the Nigella sativa L. seed, Int. Immunopharmacol. 5 (2005) 1749-1770, PMID: 16275613.

[4] K.M. Alkharfy, N.M. Al-Daghri, O.S. Al-Attas, M.S. Alokail, The protective effect of thymoquinone against sepsis syndrome morbidity and mortality in mice, Int. Immunopharmacol. 11 (2011) 250254, DOI: 10.1016/j.intimp.2010.11.032, PMID: 21145996.

[5] K.H. Khalife, G. Lupidi, Nonenzymatic reduction of thymoquinone in physiological conditions, Free Radical Res. 41 (2007) 153-161, DOI: 10.1080/10715760600978815 